\title{
Fuzzy Connections and Relations in Complete Residuated Lattices
}

Yong Chan Kim

Department of Mathematics, Gangneung-Wonju National University, Gangneung, Korea

\section{]jfis}

Received: Feb. 1, 2013

Revised : Sep. 3, 2013

Accepted: Sep. 18, 2013

Correspondence to: Yong Chan Kim (yck@gwnu.ac.kr)

(CThe Korean Institute of Intelligent Systems

(c) This is an Open Access article distributed under the terms of the Creative Commons Attribution Non-Commercial License (http://creativecommons.org/licenses/ by-nc/3.0// which permits unrestricted noncommercial use, distribution, and reproduction in any medium, provided the original work is properly cited.

\begin{abstract}
In this paper, we investigate the properties of fuzzy Galois (dual Galois, residuated, and dual residuated) connections in a complete residuated lattice $L$. We give their examples. In particular, we study fuzzy Galois (dual Galois, residuated, dual residuated) connections induced by $L$-fuzzy relations.
\end{abstract}

Keywords: Fuzzy Galois connections, Fuzzy posets, Fuzzy isotone maps, Fuzzy antitone maps

\section{Introduction}

A Galois connection is an important mathematical tool for algebraic structure, data analysis, and knowledge processing [1-10]. Hájek [11] introduced a complete residuated lattice $L$ that is an algebraic structure for many-valued logic. A context consists of $(U, V, R)$, where $U$ is a set of objects, $V$ is a set of attributes, and $R$ is a relation between $U$ and $V$. Bělohlávek [1-3] developed a notion of fuzzy contexts using Galois connections with $R \in L^{X \times Y}$ on $L$.

In this paper, we investigate properties of fuzzy Galois (dual Galois, residuated, and dual residuated) connections in $L$ and give their examples. In particular, we study fuzzy Galois (dual Galois, residuated, and dual residuated) connections induced by $L$-fuzzy relations.

Definition 1.1. $[11,12]$ An algebra $(L, \wedge, \vee, \odot, \rightarrow, 0,1)$ is called a complete residuated lattice if it satisfies the following conditions:

(C1) $L=(L, \leq, \vee, \wedge, 1,0)$ is a complete lattice with the greatest element 1 and the least element 0 ;

(C2) $(L, \odot, 1)$ is a commutative monoid;

(C3) $x \odot y \leq z$ iff $x \leq y \rightarrow z$ for $x, y, z \in L$.

Remark 1.2. [11, 12] (1) A completely distributive lattice $L=(L, \leq, \vee, \wedge=\odot, \rightarrow, 1,0)$ is a complete residuated lattice defined by

$$
x \rightarrow y=\bigvee\{z \mid x \wedge z \leq y\}
$$

In particular, the unit interval $([0,1], \vee, \wedge=\odot, \rightarrow, 0,1)$ is a complete residuated lattice defined by

$$
x \rightarrow y=\bigvee\{z \mid x \wedge z \leq y\} .
$$

(2) The unit interval with a left-continuous t-norm $\odot,([0,1], \vee, \wedge, \odot, \rightarrow, 0,1)$, is a complete residuated lattice defined by 


$$
x \rightarrow y=\bigvee\{z \mid x \odot z \leq y\}
$$

In this paper, we assume that $(L, \wedge, \vee, \odot, \rightarrow, 0,1)$ is a complete residuated lattice with the law of double negation, i.e., $a=a^{* *}$ where $a=a \rightarrow 0$.

Lemma 1.3. [12] For each $x, y, z, x_{i}, y_{i} \in L$, we have the following properties.

(1) If $y \leq z,(x \odot y) \leq(x \odot z), x \rightarrow y \leq x \rightarrow z$, and $z \rightarrow x \leq y \rightarrow x$.

(2) $x \odot y \leq x \wedge y \leq x \vee y$.

(3) $x \rightarrow\left(\bigwedge_{i \in \Gamma} y_{i}\right)=\bigwedge_{i \in \Gamma}\left(x \rightarrow y_{i}\right)$ and $\left(\bigvee_{i \in \Gamma} x_{i}\right) \rightarrow y=$ $\bigwedge_{i \in \Gamma}\left(x_{i} \rightarrow y\right)$.

(4) $x \rightarrow\left(\bigvee_{i \in \Gamma} y_{i}\right) \geq \bigvee_{i \in \Gamma}\left(x \rightarrow y_{i}\right)$.

(5) $\left(\bigwedge_{i \in \Gamma} x_{i}\right) \rightarrow y \geq \bigvee_{i \in \Gamma}\left(x_{i} \rightarrow y\right)$.

(6) $(x \odot y) \rightarrow z=x \rightarrow(y \rightarrow z)=y \rightarrow(x \rightarrow z)$.

(7) $x \odot(x \rightarrow y) \leq y, x \rightarrow y \leq(y \rightarrow z) \rightarrow(x \rightarrow z)$, and $x \rightarrow y \leq(z \rightarrow x) \rightarrow(z \rightarrow y)$.

(8) $y \leq x \rightarrow(x \odot y)$ and $x \leq(x \rightarrow y) \rightarrow y$.

(9) $x \rightarrow y \leq(x \odot z) \rightarrow(y \odot z)$.

(10) $(x \rightarrow y) \odot(y \rightarrow z) \leq x \rightarrow z$.

(11) $x \rightarrow y=1$ iff $x \leq y$.

(12) $x \rightarrow y=y^{*} \rightarrow x^{*}$.

(13) $(x \odot y)^{*}=x \rightarrow y^{*}=y \rightarrow x^{*}$ and $x \rightarrow y=\left(x \odot y^{*}\right)^{*}$.

(14) $\bigwedge_{i \in \Gamma} x_{i}^{*}=\left(\bigvee_{i \in \Gamma} x_{i}\right)^{*}$ and $\bigvee_{i \in \Gamma} x_{i}^{*}=\left(\bigwedge_{i \in \Gamma} x_{i}\right)^{*}$.

Definition 1.4. $[4,7]$ Let $X$ denote a set. A function $e_{X}$ : $X \times X \rightarrow L$ is called:

(E1) reflexive if $e_{X}(x, x)=1$ for all $x \in X$,

(E2) transitive if $e_{X}(x, y) \odot e_{X}(y, z) \leq e_{X}(x, z)$, for all $x, y, z \in X$, and

(E3) if $e_{X}(x, y)=e_{X}(y, x)=1$, then $x=y$.

If $e$ satisfies (E1) and (E2), $\left(X, e_{X}\right)$ is a fuzzy preorder set. If $e$ satisfies (E1), (E2), and (E3), $\left(X, e_{X}\right)$ is a fuzzy partially order set (for simplicity, fuzzy poset).

Example 1.5. (1) We define a function

$$
e_{L^{X}}: L^{X} \times L^{X} \rightarrow L
$$

as

$$
e_{L^{X}}(A, B)=\bigwedge_{x \in X}(A(x) \rightarrow B(x))
$$

Then, $\left(L^{X}, e_{L^{X}}\right)$ is a fuzzy poset from Lemma $1.3(10$, 11).

(2) If $\left(X, e_{X}\right)$ is a fuzzy poset and we define a function $e_{X}^{-1}(x, y)=e_{X}(y, x)$, then $\left(X, e_{X}^{-1}\right)$ is a fuzzy poset.

\section{Fuzzy Connections and Relations in Complete Residuated Lattices}

Definition 2.1. Let $\left(X, e_{X}\right)$ and $\left(Y, e_{Y}\right)$ denote fuzzy posets and $f: X \rightarrow Y$ and $g: Y \rightarrow X$ denote maps.

(1) $\left(e_{X}, f, g, e_{Y}\right)$ is called a Galois connection if for all $x \in$ $X, y \in Y$,

$$
e_{Y}(y, f(x))=e_{X}(x, g(y)) .
$$

(2) $\left(e_{X}, f, g, e_{Y}\right)$ is called a dual Galois connection if for all $x \in X, y \in Y$,

$$
e_{Y}(f(x), y)=e_{X}(g(y), x)
$$

(3) $\left(e_{X}, f, g, e_{Y}\right)$ is called a residuated connection if for all $x \in X, y \in Y$,

$$
e_{Y}(f(x), y)=e_{X}(x, g(y))
$$

(4) $\left(e_{X}, f, g, e_{Y}\right)$ is called a dual residuated connection if for all $x \in X, y \in Y$,

$$
e_{Y}(y, f(x))=e_{X}(g(y), x) \text {. }
$$

(5) $f$ is an isotone map if $e_{Y}\left(f\left(x_{1}\right), f\left(x_{2}\right)\right) \geq e_{X}\left(x_{1}, x_{2}\right)$ for all $x_{1}, x_{2} \in X$.

(6) $f$ is an antitone map if $e_{Y}\left(f\left(x_{1}\right), f\left(x_{2}\right)\right) \geq e_{X}\left(x_{2}, x_{1}\right)$ for all $x_{1}, x_{2} \in X$.

(7) $f$ is an embedding map if $e_{Y}\left(f\left(x_{1}\right), f\left(x_{2}\right)\right)=e_{X}\left(x_{1}, x_{2}\right)$ for all $x_{1}, x_{2} \in X$.

If $X=Y$ and $e_{X}=e_{Y}$, we simply denote $\left(e_{X}, f, g\right)$ for $\left(e_{X}, f, g, e_{Y}\right) .\left(X,\left(e_{X}, f, g\right)\right)$ is called a Galois (resp. residuated, dual Galois, and dual residuated) pair.

Remark 2.2. Let $\left(X, e_{X}\right)$ and $\left(Y, e_{Y}\right)$ denote a fuzzy poset and $f: X \rightarrow Y$ and $g: Y \rightarrow X$ denote maps.

(1) $\left(e_{X}, f, g, e_{Y}\right)$ is a Galois (resp. dual Galois) connection iff $\left(e_{Y}, g, f, e_{X}\right)$ is a Galois (resp. dual Galois) connection. 
(2) $\left(e_{X}, f, g, e_{Y}\right)$ is a Galois (resp. residuated) connection iff $\left(e_{X}^{-1}, f, g, e_{Y}^{-1}\right)$ is a dual (resp. dual residuated) Galois connection.

(3) $\left(e_{X}, f, g, e_{Y}\right)$ is a residuated (resp. dual residuated) connection iff $\left(e_{Y}^{-1}, g, f, e_{X}^{-1}\right)$ is a residuated (resp. dual residuated) connection.

(4) $\left(e_{X}, f, g, e_{Y}\right)$ is a Galois (resp. dual Galois) connection iff $\left(e_{X}, f, g, e_{Y}^{-1}\right)$ is a residuated (resp. dual residuated) connection.

(5) $\left(e_{X}, f, g, e_{Y}\right)$ is a residuated connection iff $\left(e_{Y}, g, f, e_{X}\right)$ is a dual residuated connection.

Theorem 2.3. Let $\left(X, e_{X}\right)$ and $\left(Y, e_{Y}\right)$ denote a fuzzy poset and $f: X \rightarrow Y$ and $g: Y \rightarrow X$ denote maps.

(1) $\left(e_{X}, f, g, e_{Y}\right)$ is a Galois connection if $f, g$ are antitone maps and $e_{Y}(y, f(g(y)))=e_{X}(x, g(f(x)))=1$.

(2) $\left(e_{X}, f, g, e_{Y}\right)$ is a dual Galois connection if $f, g$ are antitone maps and $e_{Y}(f(g(y)), y)=e_{X}(g(f(x)), x)=1$.

(3) $\left(e_{X}, f, g, e_{Y}\right)$ is a residuated connection if $f, g$ are isotone maps and $e_{Y}(f(g(y)), y)=e_{X}(x, g(f(x)))=1$.

(4) $\left(e_{X}, f, g, e_{Y}\right)$ is a dual residuated connection if $f, g$ are isotone maps and $e_{Y}(y, f(g(y)))=e_{X}(g(f(x)), x)=$ 1.

Proof. (1) Let $(f, g)$ denote a Galois connection. Since

$$
e_{Y}(y, f(x))=e_{X}(x, g(y))
$$

we have

$$
1=e_{Y}(f(x), f(x))=e_{X}(x, g(f(x)))
$$

and

$$
e_{Y}(y, f(g(y)))=e_{X}(g(y), g(y))=1 \text {. }
$$

Furthermore,

$$
\begin{aligned}
e_{Y}\left(f\left(x_{1}\right), f\left(x_{2}\right)\right) & =e_{X}\left(x_{2}, g\left(f\left(x_{1}\right)\right)\right) \\
& \geq e_{X}\left(x_{2}, x_{1}\right) \odot e_{X}\left(x_{1}, g\left(f\left(x_{1}\right)\right)\right) \\
& =e_{X}\left(x_{2}, x_{1}\right) . \\
e_{X}\left(g\left(y_{1}\right), g\left(y_{2}\right)\right) & =e_{Y}\left(y_{2}, f\left(g\left(y_{1}\right)\right)\right) \\
& \geq e_{Y}\left(y_{2}, y_{1}\right) \odot e_{Y}\left(y_{1}, f\left(g\left(y_{1}\right)\right)\right) \\
& =e_{Y}\left(y_{2}, y_{1}\right) .
\end{aligned}
$$

Conversely,

$$
\begin{aligned}
e_{Y}(y, f(x)) & \geq e_{Y}\left(y, f(g(y)) \odot e_{Y}(f(g(y)), f(x))\right. \\
& =e_{Y}(f(g(y)), f(x)) \\
& \geq e_{X}(x, g(y)) .
\end{aligned}
$$

Similarly, $e_{Y}(y, f(x)) \leq e_{X}(x, g(y))$.

(2) Since $e_{Y}(f(x), y)=e_{X}(g(y), x)$, we have

$$
1=e_{Y}(f(x), f(x))=e_{X}(g(f(x)), x)
$$

and

$$
e_{Y}(f(g(y)), y)=e_{X}(g(y), g(y))=1 .
$$

Furthermore,

$$
\begin{aligned}
e_{Y}\left(f\left(x_{1}\right), f\left(x_{2}\right)\right) & =e_{X}\left(g\left(f\left(x_{2}\right)\right), x_{1}\right) \\
& \geq e_{X}\left(x_{2}, x_{1}\right) \odot e_{X}\left(g\left(f\left(x_{2}\right)\right), x_{2}\right) \\
& =e_{X}\left(x_{2}, x_{1}\right) . \\
e_{X}\left(g\left(y_{1}\right), g\left(y_{2}\right)\right) & =e_{X}\left(f\left(g\left(y_{2}\right)\right), y_{1}\right) \\
& \geq e_{Y}\left(y_{2}, y_{1}\right) \odot e_{Y}\left(f\left(g\left(y_{2}\right)\right), y_{2}\right) \\
& =e_{Y}\left(y_{2}, y_{1}\right) .
\end{aligned}
$$

Conversely,

$$
\begin{aligned}
e_{Y}(f(x), y) & \geq e_{Y}\left(f(x), f(g(y)) \odot e_{Y}(f(g(y)), y)\right. \\
& =e_{Y}(f(x), f(g(y))) \\
& \geq e_{X}(g(y), x) .
\end{aligned}
$$

Similarly, $e_{Y}(f(x), y) \leq e_{X}(g(y), x)$

(3) Since $e_{Y}(f(x), y)=e_{X}(x, g(y))$, we have

$$
1=e_{Y}(f(x), f(x))=e_{X}(x, g(f(x)))
$$

and

$$
e_{Y}(f(g(y)), y)=e_{X}(g(y), g(y))=1 \text {. }
$$

Furthermore,

$$
\begin{aligned}
e_{Y}\left(f\left(x_{1}\right), f\left(x_{2}\right)\right) & =e_{X}\left(x_{1}, g\left(f\left(x_{2}\right)\right)\right) \\
& \geq e_{X}\left(x_{1}, x_{2}\right) \odot e_{X}\left(x_{2}, g\left(f\left(x_{2}\right)\right)\right) \\
& =e_{X}\left(x_{1}, x_{2}\right) . \\
e_{X}\left(g\left(y_{1}\right), g\left(y_{2}\right)\right) & =e_{X}\left(f\left(g\left(y_{1}\right)\right), y_{2}\right) \\
& \geq e_{Y}\left(y_{1}, y_{2}\right) \odot e_{Y}\left(f\left(g\left(y_{1}\right)\right), y_{1}\right) \\
& =e_{Y}\left(y_{1}, y_{2}\right) .
\end{aligned}
$$


Conversely,

$$
\begin{aligned}
e_{Y}(f(x), y) & \geq e_{Y}\left(f(x), f(g(y)) \odot e_{Y}(f(g(y)), y)\right. \\
& =e_{Y}(f(x), f(g(y))) \\
& \geq e_{X}(x, g(y))
\end{aligned}
$$

Moreover,

$$
\begin{aligned}
e_{X}(x, g(y)) & \geq e_{X}(x, g(f(x))) \odot e_{X}(g(f(x)), g(y)) \\
& =e_{X}(g(f(x)), g(y)) \\
& \geq e_{Y}(f(x), y) .
\end{aligned}
$$

(4) It is similarly proved as (3).

Example 2.4. Let $X=\{a, b, c\}$ denote a set and $f: X \rightarrow X$ denote a function as $f(a)=b, f(b)=a, f(c)=c$. Define a binary operation $\odot$ (called Łukasiewicz conjunction) on $L=$ $[0,1]$ using

$$
\begin{aligned}
& x \odot y=\max \{0, x+y-1\}, \\
& x \rightarrow y=\min \{1-x+y, 1\} .
\end{aligned}
$$

(1) Let $\left(X=\{a, b, c\}, e_{1}\right)$ denote a fuzzy poset as follows:

$$
e_{1}=\left(\begin{array}{cccc} 
& a & b & c \\
a & 1.0 & 0.6 & 0.5 \\
b & 0.6 & 1.0 & 0.5 \\
c & 0.7 & 0.7 & 1.0
\end{array}\right)
$$

Since $e_{1}(x, y)=e_{1}(f(x), f(y))$,

$$
e_{1}(x, f(f(x)))=e_{1}(f(f(x)), x)=1,
$$

then, $\left(e_{1}, f, f\right)$ are both residuated and dual residuated connections. Since $0.7=e_{1}(c, a) \not \leq e_{1}(f(a), f(c))=$ $e_{1}(b, c)=0.5, f$ is not an antitone map. Hence, $\left(e_{1}, f, f\right)$ are neither Galois nor dual Galois connections.

(2) Let $\left(X=\{a, b, c\}, e_{2}\right)$ denote a fuzzy poset as follows:

$$
e_{2}=\left(\begin{array}{cccc} 
& a & b & c \\
a & 1.0 & 0.6 & 0.5 \\
b & 0.6 & 1.0 & 0.7 \\
c & 0.7 & 0.5 & 1.0
\end{array}\right)
$$

Since $e_{2}(x, y)=e_{2}(f(y), f(x))$,

$$
e_{2}(x, f(f(x)))=e_{2}(f(f(x)), x)=1,
$$

then, $\left(e_{2}, f, f\right)$ are both Galois and dual Galois con- nections. Since $0.7=e_{2}(c, a) \not \leq e_{2}(f(c), f(a))=$ $e_{2}(c, b)=0.5, f$ is not an isotone map. Hence, $\left(e_{2}, f, f\right)$ are neither residuated nor dual residuated connections.

Definition 2.5. Let $R \in L^{X \times Y}$ denote a fuzzy relation. For each $A \in L^{X}$ and $B \in L^{Y}$, we define operations $R^{-1}(y, x)=$ $R(x, y)$ and $\mathcal{F}, \mathcal{G}_{R}, \mathcal{H}_{R}, \mathcal{I}_{R}, \mathcal{J}_{R}, \mathcal{K}_{R}: L^{Y} \rightarrow L^{X}$ as follows:

$$
\begin{aligned}
& \mathcal{F}_{R}(B)(x)=\bigwedge_{y \in Y}(R(x, y) \rightarrow B(y)), \\
& \mathcal{G}_{R}(B)(x)=\bigwedge_{y \in Y}(B(y) \rightarrow R(x, y)), \\
& \mathcal{H}_{R}(B)(x)=\bigvee_{y \in V}(R(x, y) \odot B(y)), \\
& \mathcal{I}_{R}(B)(x)=\bigvee_{y \in V}\left(R^{*}(x, y) \odot B^{*}(y)\right), \\
& \mathcal{J}_{R}(B)(x)=\bigwedge_{y \in Y}\left(R(x, y) \rightarrow B^{*}(y)\right), \\
& \mathcal{K}_{R}(B)(x)=\bigvee_{y \in V}\left(R(x, y) \odot B^{*}(y)\right)
\end{aligned}
$$

Theorem 2.6. Let $R \in L^{X \times Y}$ denote a fuzzy relation. For each $A \in L^{X}$ and $B \in L^{Y}$,

(1) $\mathcal{H}_{R}(B)=\left(\mathcal{F}_{R}\left(B^{*}\right)\right)^{*}, \mathcal{I}_{R}(B)=\left(\mathcal{G}\left(B^{*}\right)\right)^{*}$.

2) $\mathcal{G}_{R}(B)=\mathcal{F}_{R^{*}}\left(B^{*}\right),\left(\mathcal{I}_{R}(B)\right)^{*}=\mathcal{F}_{R^{*}}(B)=\mathcal{G}_{R}\left(B^{*}\right)$.

(3) $\mathcal{H}_{R^{-1}}\left(\mathcal{F}_{R}(B)\right) \leq B$ and $A \leq \mathcal{F}_{R}\left(\mathcal{H}_{R^{-1}}(A)\right)$.

(4) $B \leq \mathcal{F}_{R^{-1}}\left(\mathcal{H}_{R}(B)\right)$ and $\mathcal{H}_{R}\left(\mathcal{F}_{R^{-1}}(A)\right) \leq A$.

(5) $A \leq \mathcal{G}_{R}\left(\mathcal{G}_{R^{-1}}(A)\right)$ and $B \leq \mathcal{G}_{R^{-1}}\left(\mathcal{G}_{R}(B)\right)$.

(6) $A \leq \mathcal{J}_{R}\left(\mathcal{J}_{R^{-1}}(A)\right)$ and $B \leq \mathcal{J}_{R^{-1}}\left(\mathcal{J}_{R}(B)\right)$.

(7) $\mathcal{I}_{R}\left(\mathcal{I}_{R^{-1}}(A)\right) \leq A$ and $\mathcal{I}_{R^{-1}}\left(\mathcal{I}_{R}(B)\right) \leq B$.

(8) $\mathcal{K}_{R}\left(\mathcal{K}_{R^{-1}}(A)\right) \leq A$ and $\mathcal{K}_{R^{-1}}\left(\mathcal{K}_{R}(B)\right) \leq B$.

(9) $\mathcal{F}, \mathcal{H}_{R}:\left(L^{Y}, e_{L^{Y}}\right) \rightarrow\left(L^{X}, e_{L^{X}}\right)$ are isotone maps.

(10) $\mathcal{G}_{R}, \mathcal{I}_{R}, \mathcal{J}_{R}, \mathcal{K}_{R}:\left(L^{Y}, e_{L^{Y}}\right) \rightarrow\left(L^{X}, e_{L^{X}}\right)$ are antitone maps.

Proof. (1) From Lemma $1.3(13,14)$, we have

$$
\begin{aligned}
& \left(\mathcal{F}_{R}\left(B^{*}\right)\right)^{*}(x) \\
& =\left(\bigwedge_{y \in X}\left(R(x, y) \rightarrow B^{*}(y)\right)\right)^{*} \\
& =\left(\bigwedge_{y \in X}(R(x, y) \odot B(y))^{*}\right)^{*} \\
& =\bigvee_{y \in X}(R(x, y) \odot B(y))=\mathcal{H}_{R}(B)(x) .
\end{aligned}
$$




$$
\begin{aligned}
& \left(\mathcal{G}_{R}\left(B^{*}\right)\right)^{*}(x) \\
& =\left(\bigwedge_{y \in X}\left(B^{*}(y) \rightarrow R(x, y)\right)\right)^{*} \\
& =\left(\bigwedge_{y \in X}\left(R^{*}(x, y) \odot B^{*}(y)\right)^{*}\right)^{*} \\
& =\bigvee_{y \in X}\left(R^{*}(x, y) \odot B^{*}(y)\right)=\mathcal{I}_{R}(B)(x) .
\end{aligned}
$$

(2)

$$
\begin{aligned}
& \mathcal{F}_{R^{*}}\left(B^{*}\right)(x) \\
&=\bigwedge_{y \in X}\left(R^{*}(x, y) \rightarrow B^{*}(y)\right) \text { (by Lemma 1.3 (12)) } \\
&=\bigwedge_{y \in X}(B(y) \rightarrow R(x, y))=\mathcal{G}_{R}(B)(x) . \\
&\left(\mathcal{I}_{R}(B)\right)^{*}(x) \\
&=\left(\bigvee_{y \in X}\left(R^{*}(x, y) \odot B^{*}(y)\right)^{*}\right. \\
&= \bigwedge_{y \in X}\left(R^{*}(x, y) \rightarrow B(y)\right)=\mathcal{F}_{R^{*}}(B)(x) \\
&= \bigwedge_{y \in X}\left(B^{*}(y) \rightarrow R(x, y)\right)=\mathcal{G}_{R}\left(B^{*}\right)(x) .
\end{aligned}
$$

(3) From Lemma 1.3 (7), we have

$$
\begin{aligned}
& \mathcal{H}_{R^{-1}}\left(\mathcal{F}_{R}(B)\right)(x) \\
& =\bigvee_{y \in X}\left(R^{-1}(x, y) \odot \mathcal{F}_{R}(B)(y)\right) \\
& =\bigvee_{y \in X}\left(R(y, x) \odot \bigwedge_{z \in X}(R(y, z) \rightarrow B(z))\right. \\
& \leq \bigvee_{y \in X}(R(y, x) \odot(R(y, x) \rightarrow B(x)) \leq B(x)
\end{aligned}
$$

From Lemma 1.3 (8), we have

$$
\begin{aligned}
& \mathcal{F}_{R}\left(\mathcal{H}_{R^{-1}}(A)\right)(x) \\
& =\bigwedge_{y \in X}\left(R(x, y) \rightarrow \mathcal{H}_{R^{-1}}(A)(y)\right) \\
& =\bigwedge_{y \in X}\left(R(x, y) \rightarrow \bigvee_{z \in X}\left(R^{-1}(y, z) \rightarrow A(z)\right)\right. \\
& \geq \bigwedge_{y \in X}(R(x, y) \rightarrow(R(x, y) \odot A(x)) \geq A(x)
\end{aligned}
$$

(4) From Lemma 1.3 (8), we have

$$
\begin{aligned}
& \mathcal{G}_{R}\left(\mathcal{G}_{R^{-1}}(A)\right)(x) \\
& =\bigwedge_{y \in X}\left(\mathcal{G}_{R^{-1}}(A)(y) \rightarrow R(x, y)\right) \\
& =\bigwedge_{y \in X}\left(\bigwedge_{z \in X}\left(A(z) \rightarrow R^{-1}(y, z)\right) \rightarrow R(x, y)\right) \\
& \geq \bigwedge_{y \in X}((A(x) \rightarrow R(x, y)) \rightarrow R(x, y)) \\
& \geq A(x) .
\end{aligned}
$$

(6)

$$
\begin{aligned}
& \mathcal{J}_{R}\left(\mathcal{J}_{R^{-1}}(A)\right)(x) \\
& =\bigwedge_{y \in Y}\left(R(x, y) \rightarrow\left(\mathcal{J}_{R^{-1}}(A)\right)^{*}(y)\right) \\
& =\bigwedge_{y \in Y}\left(R(x, y) \rightarrow \bigvee_{z \in X}\left(R^{*-1}(y, z) \odot A(z)\right)\right) \\
& \geq \bigwedge_{y \in Y}(R(x, y) \rightarrow(R(x, y) \odot A(x))) \\
& \geq A(x)
\end{aligned}
$$

(7)

$$
\begin{aligned}
& \mathcal{I}_{R}\left(\mathcal{I}_{R^{-1}}(A)\right)(x) \\
& =\bigvee_{y \in Y}\left(R^{*}(x, y) \odot\left(\mathcal{I}_{R^{-1}}(A)\right)^{*}(y)\right) \\
& =\bigvee_{y \in Y}\left(R^{*}(x, y) \odot \bigwedge_{z \in X}\left(R^{*-1}(y, z) \rightarrow A(z)\right)\right) \\
& \leq \bigwedge_{y \in Y}\left(R^{*}(x, y) \odot\left(R^{*-1}(y, x) \rightarrow A(x)\right)\right) \\
& \leq A(x)
\end{aligned}
$$

(9) $e_{L^{Y}}(A, B) \leq e_{L^{X}}\left(\mathcal{F}_{R}(A), \mathcal{F}_{R}(B)\right)$ from:

$$
\begin{aligned}
& \mathcal{F}_{R}(A)(x) \rightarrow \mathcal{F}_{R}(B)(x) \\
&= \bigwedge_{y \in Y}(R(x, y) \rightarrow A(y)) \rightarrow \bigwedge_{y \in Y}(R(x, y) \rightarrow B(y)) \\
& \geq \bigwedge_{y \in Y}((R(x, y) \rightarrow A(y)) \rightarrow(R(x, y) \rightarrow B(y))) \\
& \geq \bigwedge_{y \in Y}(A(y) \rightarrow B(y)) . \text { (by Lemma 1.3(7)) } \\
& e_{L^{Y}}(A, B) \leq e_{L^{X}}\left(\mathcal{H}_{R}(A), \mathcal{H}_{R}(B)\right) \text { from: } \\
& \\
& \mathcal{H}_{R}(A)(x) \rightarrow \mathcal{H}_{R}(B)(x) \\
&=\bigvee_{y \in Y}(R(x, y) \odot A(y)) \rightarrow \bigvee_{y \in Y}(R(x, y) \odot B(y)) \\
& \geq \bigwedge_{y \in Y}((R(x, y) \odot A(y)) \rightarrow(R(x, y) \odot B(y))) \\
& \geq \bigwedge_{y \in Y}(A(y) \rightarrow B(y)) .(\text { by Lemma } 1.3(9))
\end{aligned}
$$

(10) $e_{L^{Y}}(A, B) \leq e_{L^{X}}\left(\mathcal{G}_{R}(B), \mathcal{G}_{R}(A)\right)$ from:

$$
\begin{aligned}
& \mathcal{G}_{R}(B)(x) \rightarrow \mathcal{G}_{R}(A)(x) \\
&=\bigwedge_{y \in Y}(B(y) \rightarrow R(x, y)) \rightarrow \bigwedge_{y \in Y}(A(y) \rightarrow R(x, y)) \\
& \geq \bigwedge_{y \in Y}((B(y) \rightarrow R(x, y)) \rightarrow(A(y) \rightarrow R(x, y))) \\
& \geq \bigwedge_{y \in Y}(A(y) \rightarrow B(y)) \text {. (by Lemma 1.3 (7)) } \\
& e_{L^{Y}}(A, B) \leq e_{L^{X}}\left(\mathcal{I}_{R}(B), \mathcal{I}_{R}(A)\right) \text { from: } \\
& \\
& \mathcal{I}_{R}(B)(x) \rightarrow \mathcal{I}_{R}(A)(x) \\
&=\bigvee_{y \in Y}\left(R^{*}(x, y) \odot B^{*}(y)\right) \rightarrow \bigvee_{y \in Y}\left(R^{*}(x, y) \odot A^{*}(y)\right) \\
& \geq \bigwedge_{y \in Y}\left(\left(R^{*}(x, y) \odot B^{*}(y)\right) \rightarrow\left(R^{*}(x, y) \odot A^{*}(y)\right)\right) \\
& \geq \bigwedge_{y \in Y}\left(B^{*}(y) \rightarrow A^{*}(y)\right)(\text { by Lemma } 1.3(9)) \\
& \geq \bigwedge_{y \in Y}(A(y) \rightarrow B(y)) .(\text { by Lemma } 1.3(12))
\end{aligned}
$$

Similarly, $e_{L^{Y}}(A, B) \leq e_{L^{X}}\left(\mathcal{K}_{R}(B), \mathcal{K}_{R}(A)\right)$. $e_{L^{Y}}(A, B) \leq e_{L^{X}}\left(\mathcal{J}_{R}(B), \mathcal{J}_{R}(A)\right)$ from:

$$
\begin{aligned}
& \mathcal{J}_{R}(B)(x) \rightarrow \mathcal{J}_{R}(A)(x) \\
& =\bigwedge_{y \in Y}\left(R(x, y) \rightarrow B^{*}(y)\right) \rightarrow \bigwedge_{y \in Y}\left(R(x, y) \rightarrow A^{*}(y)\right) \\
& \geq \bigwedge_{y \in Y}\left(\left(R(x, y) \rightarrow B^{*}(y)\right) \rightarrow\left(R(x, y) \rightarrow A^{*}(y)\right)\right) \\
& \geq \bigwedge_{y \in Y}\left(B^{*}(y) \rightarrow A^{*}(y)\right) \text { (by Lemma 1.3 (9)) } \\
& \geq \bigwedge_{y \in Y}(A(y) \rightarrow B(y)) \text {. (by Lemma 1.3 (7)) }
\end{aligned}
$$

Other cases are similarly proved.

Theorem 2.7. Let $R \in L^{X \times Y}$ denote a fuzzy relation, $\left(L^{X}\right.$, 
$\left.e_{L^{X}}\right)$ and $\left(L^{Y}, e_{L^{Y}}\right)$ denote fuzzy posets. We have the following properties.

(1) $\left(e_{L^{Y}}, \mathcal{H}_{R}, \mathcal{F}_{R^{-1}}, e_{L^{X}}\right)$ and $\left(e_{L^{X}}, \mathcal{H}_{R^{-1}}, \mathcal{F}_{R}, e_{L^{Y}}\right)$ are residuated connections.

(2) $\left(e_{L^{Y}}, \mathcal{F}_{R}, \mathcal{H}_{R^{-1}}, e_{L^{X}}\right)$ and $\left(e_{L^{X}}, \mathcal{F}_{R^{-1}}, \mathcal{H}_{R}, e_{L^{Y}}\right)$ are dual residuated connections.

(3) $\left(e_{L^{Y}}, \mathcal{G}_{R}, \mathcal{G}_{R^{-1}}, e_{L^{X}}\right)$ and $\left(e_{L^{Y}}, \mathcal{J}_{R}, \mathcal{J}_{R^{-1}}, e_{L^{X}}\right)$ are Galois connections.

(4) $\left(e_{L^{Y}}, \mathcal{I}_{R}, \mathcal{I}_{R^{-1}}, e_{L^{X}}\right)$ and $\left(e_{L^{Y}}, \mathcal{K}_{R}, \mathcal{K}_{R^{-1}}, e_{L^{X}}\right)$ are dual Galois connections.

Proof. (1) For each $C \in L^{X}, B \in L^{Y}$,

$$
\begin{aligned}
& e_{L^{X}}\left(\mathcal{H}_{R}(B), C\right) \\
& =\bigwedge_{x \in X}\left(\mathcal{H}_{R}(B)(x) \rightarrow C(x)\right) \\
& =\bigwedge_{x \in X}\left(\bigvee_{y \in Y}(R(x, y) \odot B(y)) \rightarrow C(x)\right) \\
& =\bigwedge_{x \in X} \bigwedge_{y \in V}(B(y) \rightarrow(R(x, y) \rightarrow C(x))) \\
& =\bigwedge_{y \in Y}\left(B(y) \rightarrow \bigwedge_{x \in X}\left(R^{-1}(y, x) \rightarrow C(x)\right)\right) \\
& =\bigwedge_{y \in Y}\left(B(y) \rightarrow \mathcal{F}_{R^{-1}}(C)(y)\right) \\
& =e_{L^{Y}}\left(B, \mathcal{F}_{R^{-1}}(C)\right) .
\end{aligned}
$$

(2) For each $C \in L^{X}, B \in L^{Y}$,

$$
\begin{aligned}
& e_{L^{X}}\left(C, \mathcal{F}_{R}(B)\right) \\
& =\bigwedge_{x \in X}\left(C(x) \rightarrow \mathcal{F}_{R}(B)(x)\right) \\
& =\bigwedge_{x \in X}\left(C(x) \rightarrow \bigwedge_{y \in Y}(R(x, y) \rightarrow B(y))\right) \\
& =\bigwedge_{y \in Y} \bigwedge_{x \in X}((C(x) \odot R(x, y)) \rightarrow B(y)) \\
& =\bigwedge_{y \in Y}\left(\bigvee_{x \in X}(C(x) \odot R(x, y)) \rightarrow B(y)\right) \\
& =\bigwedge_{y \in Y}\left(\mathcal{H}_{R^{-1}}(C)(y) \rightarrow B(y)\right) \\
& =e_{L^{Y}}\left(\mathcal{H}_{R^{-1}}(C), B\right) .
\end{aligned}
$$

(3) For each $C \in L^{X}, B \in L^{Y}$,

$$
\begin{aligned}
& e_{L^{X}}\left(C, \mathcal{J}_{R}(B)\right) \\
& =\bigwedge_{x \in X}\left(C(x) \rightarrow \mathcal{J}_{R}(B)(x)\right) \\
& =\bigwedge_{x \in X}\left(C(x) \rightarrow \bigwedge_{y \in Y}\left(R(x, y) \rightarrow B^{*}(y)\right)\right) \\
& =\bigwedge_{x \in X}\left(C(x) \rightarrow \bigwedge_{y \in Y}(R(x, y) \odot B(y) \rightarrow 0)\right) \\
& =\bigwedge_{y \in Y} \bigwedge_{x \in X}((C(x) \odot R(x, y) \odot B(y)) \rightarrow 0) \\
& =\bigwedge_{y \in Y}\left(B(y) \rightarrow \bigwedge_{x \in X}((R(x, y) \odot C(x)) \rightarrow 0)\right) \\
& =\bigwedge_{y \in Y}\left(B(y) \rightarrow \bigwedge_{x \in X}\left(R^{-1}(y, x) \rightarrow C^{*}(x)\right)\right. \\
& =\bigwedge_{y \in Y}\left(B(y) \rightarrow \mathcal{J}_{R^{-1}}(C)(y)\right) \\
& =e_{L^{Y}}\left(B, \mathcal{J}_{R^{-1}}(C)\right) .
\end{aligned}
$$

(4) For each $C \in L^{X}, B \in L^{Y}$,

$$
\begin{aligned}
& e_{L^{X}}\left(\mathcal{K}_{R}(B), C\right) \\
& =\bigwedge_{x \in X}\left(\mathcal{K}_{R}(B)(x) \rightarrow C(x)\right) \\
& =\bigwedge_{x \in X}\left(\bigvee_{y \in Y} R(x, y) \odot B^{*}(y) \rightarrow C(x)\right) \\
& =\bigwedge_{x \in X} \bigwedge_{y \in Y}\left(R(x, y) \odot B^{*}(y) \odot C^{*}(x) \rightarrow 0\right) \\
& =\bigwedge_{x \in X} \bigwedge_{y \in Y}\left(R(x, y) \odot C^{*}(x) \rightarrow B(y)\right) \\
& =\bigwedge_{y \in Y}\left(\bigvee_{x \in X}\left(R^{-1}(y, x) \odot C^{*}(x)\right) \rightarrow B(y)\right) \\
& =\bigwedge_{y \in Y}\left(\mathcal{K}_{R^{-1}}(C)(y) \rightarrow B(y)\right) \\
& =e_{L^{Y}}\left(\mathcal{K}_{R^{-1}}(C), B\right) .
\end{aligned}
$$

For each $C \in L^{X}, B \in L^{Y}$,

$$
\begin{aligned}
& e_{L^{Y}}\left(\mathcal{I}_{R}(B), C\right) \\
& =\bigwedge_{x \in X}\left(\mathcal{I}_{R}(B)(x) \rightarrow C(x)\right) \\
& =\bigwedge_{x \in X}\left(\bigvee_{y \in Y}\left(R^{*}(x, y) \odot B^{*}(y) \rightarrow C(x)\right)\right. \\
& =\bigwedge_{x \in X} \bigwedge_{y \in Y}\left(R^{*}(x, y) \odot B^{*}(y) \rightarrow C(x)\right) \\
& =\bigwedge_{x \in X} \bigwedge_{y \in Y}\left(R^{*}(x, y) \odot C^{*}(x) \rightarrow B(y)\right) \\
& =\bigwedge_{y \in Y}\left(\bigvee_{x \in X}\left(\left(R^{-1}\right)^{*}(y, x) \odot C^{*}(x)\right) \rightarrow B(y)\right) \\
& =\bigwedge_{y \in Y}\left(\mathcal{I}_{R^{-1}}(C)(y) \rightarrow B(y)\right) \\
& =e_{L^{Y}}\left(\mathcal{I}_{R^{-1}}(C), B\right) .
\end{aligned}
$$

Other cases are similarly proved.

\section{Conclusion}

In this paper, we investigated the properties of fuzzy Galois (dual Galois, residuated, and dual residuated) connections in a complete residuated lattice $L$. In particular, we studied fuzzy Galois (dual Galois, residuated, and dual residuated) connections induced by $L$-fuzzy relations.

In the future, we will investigate the properties using fuzzy connections on algebraic structures and study the fuzzy concept lattices.

\section{Conflict of Interest}

No potential conflict of interest relevant to this article was reported. 


\section{References}

[1] R. Bělohlávek, "Fuzzy Galois connections," Mathematical Logic Quarterly, vol. 45, no. 4, pp. 497-504, 1999. http://dx.doi.org/10.1002/malq.19990450408

[2] R. Bělohlávek, "Lattices of fixed points of fuzzy Galois connections," Mathematical Logic Quarterly, vol. 47, no. 1, pp. 111-116, Jan. 2001. http://dx.doi.org/10.1002/1521-3870(200101)47: 1〈111::AID-MALQ111〉3.0.CO;2-A

[3] R. Bělohlávek, "Concept lattices and order in fuzzy logic," Annals of Pure and Applied Logic, vol. 128, no. 1-3, pp. 277-298, Aug. 2004. http://dx.doi.org/10.1016/j.apal. 2003.01.001

[4] J. G. García, I. Mardones-Prez, M. A. de Prada Vicente, and D. Zhang, "Fuzzy Galois connections categorically," Mathematical Logic Quarterly, vol. 56, no. 2, pp. 131-147, Mar. 2010. http://dx.doi.org/10.1002/malq.200810044

[5] G. Georgescu and A. Popescu, "Non-dual fuzzy connections," Archive for Mathematical Logic, vol. 43, no. 8, pp. 1009-1039, Nov. 2004. http://dx.doi.org/10.1007/ s00153-004-0240-4

[6] G. Georgescu and A. Popescu, "Non-commutative fuzzy Galois connections," Soft Computing, vol. 7, no. 7, pp. 458-467, Jun. 2003. http://dx.doi.org/10.1007/ s00500-003-0280-4

[7] H. Lai and D. Zhang, "Concept lattices of fuzzy contexts: formal concept analysis vs. rough set theory," International Journal of Approximate Reasoning, vol. 50, no. 5, pp. 695-707, May 2009. http://dx.doi.org/10.1016/j.ijar. 2008.12.002
[8] A. Melton, D. A. Schmidt, and G. E. Strecker, "Galois connections and computer science applications," in Category Theory and Computer Programming, Lecture Notes in Computer Science vol. 240, D. Pitt, S. Abramsky, A. Poign, and D. Rydeheard, Eds. Heidelberg: Springer Berlin, 1986, pp. 299-312. http://dx.doi.org/10.1007/ 3-540-17162-2_130

[9] R. Wille, "Restructuring lattice theory: an approach based on hierarchies of concepts," in Proceedings of the NATO Advanced Study Institute held at Banff, Canada, August 28 to September 12, 1981, NATO Advanced Study Institutes Series vol. 83, I. Rival, Ed. The Netherlands: Springer Netherlands, 1982, pp. 445-470. http://dx.doi. org/10.1007/978-94-009-7798-3_15

[10] W. Yao and L. X. Lu, "Fuzzy Galois connections on fuzzy posets," Mathematical Logic Quarterly, vol. 55, no. 1, pp. 105-112, Jan. 2009. http://dx.doi.org/10.1002/malq. 200710079

[11] P. Hájek, Metamathematics of Fuzzy Logic, Dordrecht: Kluwer, 1998.

[12] E. Turunen, Mathematics Behind Fuzzy Logic, Heidelberg: Physica-Verlag, 1999.

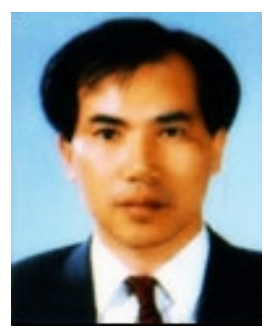

Yong Chan Kim received the B.S., M.S., and $\mathrm{Ph} . \mathrm{D}$. degrees in Mathematics from Yonsei University, Seoul, Korea, in 1982 , 1984, and 1991, respectively. He is currently Professor of Gangneung-Wonju University. His research interests include fuzzy topology and fuzzy logic.

E-mail: yck@gwnu.ac.kr 